\title{
Dilute Solution Theory of Polymer Crystal Growth: Fractionation Effects
}

\author{
Isaac C. Sanchez* and Edmund A. DiMarzio
}

\author{
Institute for Materials Research, National Bureau of Standards, Washington, D.C. 20234
}

(February 4, 1972)

\begin{abstract}
A nonequilibrium (kinetic) theory of polymer molecular weight (MW) fractionation is formulated and applied to binary and multicomponent polyethylenes crystallized from unstirred xylene solutions. High MW components crystallize more readily than do low MW components. This fractionation effect is enhanced as the crystallization temperature is raised. At low crystallization temperatures (high undercoolings) the polymer tends to fractionate according to the volume fraction distribution of its MW components and thus, the number average MW of the crystal that is formed is approximately equal to the weight average MW of the polymer in solution. It is shown that MW fractionation does not depend on the details of nucleation, but rather on the rates for post-nucleation crystal growth. The effects of MW polydispersity on crystalline properties is considered and in particular it is shown that polydispersity tends to mask the intrinsic dependence of crystal thickness on MW. The variables which govern and influence fractionation are also discussed.
\end{abstract}

Key words: Cilia; dissolution temperature; fractionation; rate constant; undercooling.

\section{Introduction}

There is a growing body of experimental evidence that indicates that when a polymer polydispersed in molecular weight (MW) begins crystallizing, the high MW components preferentially crystallize first. This phenomena has been observed in both stirred $[1-3]^{1}$ and unstirred $[1,4,5]$ solutions of polyethylene and xylene. It is also thought to occur during crystallization from the melt and would appear to affect the resulting crystalline morphologv. Fractionation has been invoked to explain spherulitic growth habits $[6,7]$ and is believed to accompany the formation of extended-chain crystals [8-12]. For monomeric materials, crystallization from solution or from the melt (zone refining) is a standard procedure for achieving high purity and one wonders what the limitations are for polymeric materials.

Polymer MW fractionation during crystal growth is also of interest for other reasons; an important one is the fact that all polymers are polydisperse in MW to some degree. For example, in crystallization studies one sometimes tries to follow the variation of a crystal property with MW (average) by working with sharp MW fractions. A knowledge of the amount of fractionation that is occurring gives one a better idea of whether the distribution is sharp enough for the experiment in question; i.e., in crystallization experiments

\footnotetext{
*NRC-NAS Postdoctoral Research Associate, 1969-71. Present address: Xerox Cor poration, Rochester, N.Y. 14603

1 Figures in brackets indicate the literature references at the end of this paper.
}

the very notion of a "sharp" MW fraction is intimately tied up with the amount of fractionation that occurs.

Moreover, MW fractionation implies that the central regions of a cyrstallite can have a MW composition that is different than the peripheral regions since the solution becomes depleted of high MW components as the crystal grows in lateral size. Thus, there is a composition heterogeneity in the crystal and a concomitant spatial variation in all those properties that are a function of composition. It has been suggested that isothermal thickening of polymer crystals is caused by this variation in crystal composition $[13,14]$.

Heretofore, theoretical studies of MW fractionation have essentially been equilibrium theories $[4,5,15,16]$. However, equilibrium treatments of fractionation have a limited range of applicability [15] since polymer crystals are formed under nonequilibrium conditions. The undercooling is typically 20 to $30{ }^{\circ} \mathrm{C}$ and as will be demonstrated, fractionation is a sensitive function of undercooling.

In a previous publication [17], hereafter referred to as paper I, we developed a kinetic theory of polymer crystal growth from dilute solution for a monodisperse polymer. In a second paper [18], paper II, we applied the theory to polyethylene (PE) crystallized from xylene solutions. These works provide a foundation on which we can build a nonequilibrium theory of fractionation in unstirred solutions. This is done in section 2 . In section 3 we apply the theory to the PE-xylene system and in section 4 the conclusions of the study are discussed. 


\section{Theory}

\subsection{Relation to Papers I and II}

It is perhaps best to begin by recalling the experimental situation on which our model is based. In dilute solution, thin (ca. $100 \AA$ ) plate-like lamellar crystals grow to large lateral size by accretion of the polymer molecules to the outer edges of the crystal. The polymer molecules composing the crystals are oriented with their molecular axes perpendicular to the basal plane of the lamella $[19,20]$. Since the contour length of the molecule is larger than the lamellar thickness the molecules must fold. Cleavage experiments show that the molecules tend to fold parallel to the growing edge [21] and crystallographic properties indicate more or less tight folds on the surface of the crystal [20]. The molecules can thus be viewed as lying down in parallel growth strips (fold planes). The growth rate is of the order of strips per second [22]. The smoothness of the growing edge is a measure of the rate of nucleating a strip compared to the rate of filling in the nucleated strip. Smooth surfaces correspond to rare nucleation events, but one cannot make a quantitative statement without recourse to theory.

In papers I and II we described and calculated the growth rates of such crystals from a monodisperse polymer solution. One of the interesting aspects of this treatment is that the rate of nucleation $S^{*}$ of a strip is a rare event compared to the rate of filling in of the strip, $S^{\prime}$, under most growth conditions. This means that the overwhelming number of molecules that comprise the grown strip, and therefore the crystal, are non-nucleating molecules. Under these conditions one can write the growth rate $G$ for the change of a lateral dimension with time as [17]

$$
G=G\left(S^{*}, S^{\prime}, N\right)
$$

where $N$ is the number of nucleation sites. In the more general case $G$ is a function of the forward and backward rate constants for nucleation as well as the forward and backward rate constants for filling in.

This formula remains valid even if we have a polydisperse system, the $S^{*}$ and $S^{\prime}$ merely need be interpreted as total growth rates:

$$
\begin{aligned}
& S^{*}=\sum S_{i}^{*} \\
& S^{\prime}=\sum S_{i}^{\prime}
\end{aligned}
$$

$S_{i}^{\prime}$ is the net rate at which molecules of type $i$ are being added to an already nucleated strip. Since most molecules of a growth strip are of the fill-in type the fraction of species $i$ incorporated into the crystal is given by

$$
f_{i}=S_{i}^{\prime} / \sum S_{i}^{\prime}
$$

Nucleation must be viewed as the compound event of attaching a molecule (or cilium) to the crystal edge followed by the subsequent stabilization of this molecule by other molecules. $S_{i}^{*}$ is the rate at which molecules of type $i$ initiate the growth of a strip. The fraction of growth strips whose initial molecule is type $i$ is a calculable quantity but it is uninteresting.

In paper I we pointed out that the functional form of eq (2.1) for the growth rate $G$ is unknown, except in two limiting cases. We argued that for polymer crystallization the following limiting case

$$
G \sim S^{*}
$$

is valid when $S^{*} \ll S^{\prime}$. We wish to point out that the composition of the crystal does not depend on the functional form of $G$ and the prescription for calculating the composition remains valid even if $S^{*}$ is sufficiently close to $S^{\prime}$ so that eq $(2.5)$ is no longer a useful approximation. Hillig has given an alternate expression for $G$ which might be a useful approximation in some regions of growth [23]. His method can be easily adapted to our two dimensional growth problem. The result is

$$
G \sim\left(S^{*} S^{\prime}\right)^{1 / 2} .
$$

Equation (2.5) is expected to hold when the number of nuclei per $N$ sites is the order of one or less (i.e., $\left.S^{\prime} / S^{*} N>1\right)$ and results in a smooth edge, while eq (2.6) holds when $S^{\prime} / S^{*} N$ is small and results in a rough (highly reentrant growth) edge. As was pointed out in the appendix of paper I the effective value of $N$ may be considerably smaller than the number of sites on an edge due to defects of various types which destroy the continuity of the crystal along the edge.

\subsection{LDP Kinetic Theory}

Lauritzen, DiMarzio, and Passaglia (LDP) have formulated a theory to describe the steady state rate of growth and composition of a chain growing in a multicomponent system [24]. A chain in the system grows by the sequential addition of any of the $n$ components (species) of the system to one end of the chain. Only nearest neighbor interactions are considered; the rate constants $\alpha^{i j}$ for adding species $j$ to a chain ending in species $i$ and $\beta^{i j}$ for removing species $j$ from a chain ending in $(i, j)$ are assumed to be independent of chain length (this restriction can be relaxed, [25] but it is not necessary to do so in the present application of the theory). Initiation of a new chain by species $j$ is governed by the unique rate constant pair $\left(\alpha_{0}^{j}, \beta_{1}^{j}\right)$.

If $S_{\nu}^{j}$ is the current or rate at which chains $\nu$ units long ending with species $j$ are produced, $S_{\nu}^{i j}$ the current for chains $\nu$ units long ending with the pair $(i, j)$ etc., then LDP showed that

$$
\lim _{\nu \rightarrow \infty} S_{\nu}^{j} / N_{\nu}^{j}=S_{\nu}^{i j} / N_{\nu}^{i j}=\ldots=\lambda^{j}
$$

where $N_{\nu}^{j}$ is the number of chains (occupation number) $\nu$ units long that end with species $j$ in the ensemble of chains and $N_{v}^{i j}$ and higher order occupation numbers are similarly defined. The ratios of currents to their corresponding occupation number in the limit of large chain lengths become a function of the terminal 
species only. Also the currents and occupation numbers become independent of $\nu$ for large $\nu$. The quantities $\lambda^{j}$ are defined by a set of nonlinear equations:

$$
\lambda^{j}=\sum_{i=1}^{n} \frac{\alpha^{j i} \lambda^{i}}{\beta^{j i}+\lambda^{i}}
$$

where $n$ is the total number of components in the system. The net rate at which chains are initiated is given by

$$
S_{T}=\sum S^{j}=\sum_{j} \lambda^{j} N^{j}=\sum_{j} \frac{\alpha_{0}^{j} \lambda^{j} N_{0}}{\beta_{1}^{j}+\lambda^{j}}
$$

where $N_{0}$ is the number of sites where chain initiation can occur. The fraction $f_{j}$ of component $j$ in the chain is given by

$$
f_{j}=\lambda^{j} N^{j} / \Sigma_{j} \lambda^{j} N^{j}
$$

where the occupation numbers $N^{j}$ are obtained from the recursion relation

$$
N_{\nu}^{i}=\sum_{j} \frac{\alpha^{j i} N_{\nu-1}^{j}}{\beta^{j i}+\lambda^{i}}
$$

where $N_{1}^{i}=\alpha_{0}^{i} N_{0} /\left(\beta_{1}^{i}+\lambda^{i}\right)$. For large $\nu$ eq $(2.11)$ becomes

$$
N^{i}=\sum_{j} \frac{\alpha^{j i} N^{j}}{\beta^{j i}+\lambda^{i}} .
$$

In the present application of the LDP theory, the components of the system are the various molecular weight species present in a polymer heterogeneous in molecular weight and the "chain" referred to above is a growth strip on a crystal face. As we pointed out in section 2.1, polymer single crystal growth is twodimensional (2-D) in character; however, because of the relative rates of nucleation and growth the crystal grows essentially by the sequential addition of $1-D$ growth strips (fold planes). This crystal growth feature allows one to employ a 1-D theory like the LDP theory to describe the formation and composition of these growth strips.

In paper I we showed that when the crystal growth strip was composed only of polymer molecules of degree of polymerization $P_{j}$ which added one crystal stem at a time, then there emerges effective rate constants for adding the molecule as a unit. These forward and backward rate constants for the whole molecule were denoted as $A_{0}, B_{1}$ for the nucleation step and as $\mathrm{A}_{1}, B_{2}$ for post-nucleation growth. Thus, in a system containing molecules of various lengths we can assign forward and backward rate constants $\left(A_{0}^{j}, B_{1}^{j}\right)$ and $\left(A_{1}^{j}, B_{2}^{j}\right)$ to each size molecule and evaluate these rate constants as was done in paper I and paper II. This assumes that adding a molecule of length $j$ to the crystal is independent of the molecules already in the crystal, i.e.,

$$
\begin{aligned}
& \alpha^{1 j}=\alpha^{2 j}=\ldots=\alpha^{n j}=A_{1}^{j} \\
& \beta^{1 j}=\beta^{2 j}=\ldots=\beta^{n j}=B_{2}^{j} .
\end{aligned}
$$

The identification $\alpha_{0}^{j}=A_{0}^{j}$ and $\beta_{1}^{j}=B_{1}^{j}$ completes the specification of the rate constants. This set of rate constants leads to a simplification of the LDP theory equations:

$$
\lambda^{1}=\lambda^{2}=\ldots .=\lambda^{n} \equiv \lambda
$$

and

$$
\lambda=\sum_{j}^{n} \frac{A_{1}^{j} \lambda}{B_{2}^{j}+\lambda}
$$

Moreover,

$$
S_{T}=\sum_{j}^{n} \frac{A_{0}^{j} \lambda N_{0}}{B_{1}^{j}+\lambda} .
$$

At this point we should identify the nucleation rate $S^{*}$ and the post-nucleation rate $S^{\prime}$ of section 2.1 with the LDP equations so that the full physical significance of these quantities become apparent:

$$
S_{j}^{*}=\frac{A_{0}^{j} \lambda}{B_{1}^{j}+\lambda}, S^{*}=\Sigma_{j} S_{j}^{*}=S_{T} / N_{0}
$$

and

$$
S_{j}^{\prime}=\frac{A_{1}^{j} \lambda}{B_{2}^{j}+\lambda}, S^{\prime}=\Sigma_{j} S_{j}^{\prime}=\lambda .
$$

Thus, from eq (2.4) the fraction of species $j$ that is incorporated is given by

$$
f_{j}=\frac{A_{1}^{j}}{B_{2}^{j}+\lambda} .
$$

Since $\lambda$ is only a function of the post-nucleation rate constants $\left(A_{1}^{j}, B_{2}^{j}\right), f_{j}$ is independent of the nucleation rate constants $\left(A_{0}^{j}, B_{1}^{j}\right)$. Also note that eq (2.18) can be derived directly from eqs (2.10) and (2.12).

\subsection{Energetics and Thermodynamics}

\section{a. General Considerations}

The LDP kinetic theory does not require a determination of the free energy of mixing of the components in the crystalline phase as is required by equilibrium theories of the fractionation phenomena $[5,16]$. From LDP theory, we have

$$
\alpha^{i j} / \beta^{i j}=\exp \left[\left(\mu_{j}-\epsilon_{i j}\right) / k T\right]
$$


where $\mu_{j}$ is the chemical potential of species $j$ in solution and $\epsilon_{i j}$ is the interaction energy associated with adding species $j$ to a chain ending in species $i$. The free energy change $\left(\mu_{j}-\epsilon_{i j}\right)$ that is required to evaluate the rate constants does not require or presume a knowledge of the free energy change associated with the mixing of species in the chain. In fact, the net change in chemical potentials caused by the mixing is calculable from the theory. For example, in a $n$ component system where the components are similar in size and the $\epsilon_{i j}$ are not too dissimilar, the total mixing entropy would be given to an excellent approximation by

$$
\Delta S_{\text {mix }}=-k \sum_{j}^{n} N_{j} \ln \left(N_{j} / \Sigma_{i} N_{i}\right)
$$

or

$$
\Delta S_{\text {mix }} / \Sigma_{i} N_{i}=-k \Sigma_{j} f_{j} \ln f_{j}
$$

where $N_{j}$ is the number of elements in the chain of species $j$ and $f_{j}$ is the mole fraction of species $j$ in the chain. If the components vary in size, then $\ln f_{j}$ would be replaced by $\ln v_{j}$ where $v_{j}$ is the volume fraction of species $j$ in the chain. Now the change in chemical potential $\Delta \mu_{j}$ caused by mixing is given by

$$
\begin{gathered}
\Delta \mu_{j}=\frac{\partial}{\partial N_{j}}\left(-T \Delta S_{\text {mix }}\right) \\
\Delta \mu_{j}=k T \ln f_{j}
\end{gathered}
$$

where the mole fractions $f_{j}$ can be determined from eq (2.18).

In general, when the interaction energies $\epsilon_{i j}$ cannot be ignored then [26]

$$
\Delta S_{\mathrm{mix}} / \Sigma N_{i}=-k \Sigma_{i, j} f_{i j} \ln \left(f_{i j} / f_{i}\right)
$$

where $f_{i j}$ is the fraction of $(i, j)$ pairs in the chain and from LDP theory is given by

$$
f_{i j}=\alpha^{i j} \lambda^{j} N^{i} /\left(\beta^{i j}+\lambda^{j}\right) S_{T} .
$$

In the special case where there is no correlation between components, as in the present application, then $f_{i j}=f_{i} f_{j}$ and eq $(2.22)$ reduces to eq $(2.20 \mathrm{~b})$.

$$
\text { b. Evaluation of } \boldsymbol{\mu}_{j}-\boldsymbol{\epsilon}_{i j}
$$

If the free energy change $\mu_{j}-\epsilon_{i j}$ required in eq (2.19) refers to the crystallization of the entire molecule, then (ignoring for the moment surface effects)

$$
\mu_{j}-\epsilon_{i j}=\mu_{j}^{0}-\mu_{j}^{c}+R T \ln a_{j}
$$

where $\mu_{j}^{0}$ is the chemical potential of pure species $j$ in a melt at temperature $T$ (the standard state); $\mu_{j}^{c}$ in the chemical potential of species $j$ in a homogeneous crystal of species $j$ and $a_{j}$ is the activity of species $j$ in solution.
The term $R T \ln a_{j}$ is the difference in chemical potential between a pure melt of species $j$ and a polymer solution containing all species; it is given by [27]

$$
\ln a_{j}=\ln v_{2}^{j}-\left(x_{j}-1\right)+v_{2} x_{j}\left(1-1 / \bar{x}_{\eta}\right)+x_{j} \chi\left(1-v_{2}\right)^{2}
$$

where $v_{2}^{j}$ is the volume fraction of species $j$ in solution; $v_{2}=\Sigma_{j}^{n} v_{2}^{j}$ is the total volume fraction of polymer; $x_{j}$ in the ratio of the molar volume of species $j$ to that of the solvent; $\bar{x}_{\eta}$ is the number average of $x_{j}$ is the solution phase and $\chi$ is the polymer-solvent interaction parameter which in general is a function of the molecular weight of species $j$.

The term $\mu_{j}^{c}-\mu_{j}^{0}$ is the difference in chemical potential between a homogeneous melt and a homogeneous crystal of species $j$ and is given by

$$
\mu_{j}^{0}-\mu_{j}^{c}=P_{j} \Delta G_{u}^{0}(T, j)
$$

where $P_{j}$ is the degree of polymerization of species $j$ and $\Delta G_{u}^{0}(T, j)$ is the difference in free energy between a monomer unit (belonging to species $j$ ) in a melt and one embedded in a crystal. Different approximations can be employed for $\Delta G_{u}^{0}$; however, for polyethylene (PE) the following approximation is best: [18]

$$
\Delta G_{u}^{0}(T, j)=\Delta H_{u}\left(T_{m}^{0}-T\right)\left(1+T / T_{m}^{0}\right) / 2 T_{m}^{0}
$$

where $\Delta H_{u}$ is the enthalpy of fusion per mole of monomer at the equilibrium melting point $T_{m}^{0}$. Of course, $T_{m}^{0}$ is a function of molecular weight, $T_{m}^{0} \equiv T_{m}^{0}(j)$.

It is convenient to define a "psuedo" equilibrium dissolution temperature $T_{j}^{0}$ for species $j$ by setting eq (2.24) equal to zero. Using eqs (2.26) and (2.27) yields.

$$
T_{j}^{0}=T_{m}^{0}(j)\left[r+\left(1+r^{2}\right)^{1 / 2}\right]
$$

where

$$
r=R T_{m}^{0} \ln a_{j} / \Delta H_{u} P_{j}
$$

In Paper II it was shown that if the partial molar heat of mixing molten polymer with solvent was small compared to the molar heat of fusion, then eq (2.24) can be expressed as

$$
\mu_{j}-\epsilon_{i j}=P_{j}\left[\Delta G_{u}^{0}(T, j)-\frac{T}{T_{j}^{0}} \Delta G_{u}^{0}\left(T_{j}^{0}, j\right)\right]
$$

and from eq (2.27), we have

$$
\mu_{j}-\epsilon_{i j}=P_{j}\left[\Delta H_{u}\left(T_{j}^{0}-T\right)\left(1+T T_{j}^{0} / T_{m}^{0^{2}}\right) / 2 T_{j}^{0}\right] .
$$

In summary eq (2.30) represents the change in free energy associated with removing species $j$ from a 
solution containing ali species and placing it in a crystal containing only species $j$. Of course, if the polymer molecule is in a chain-folded configuration, surface free energies must be added.

If the solution consisted only of solvent and species $j$, then $T_{j}^{0}$ becomes the equilibrium dissolution temperature $T_{d}^{0}$ for the monodisperse polymer-solvent system. The "psuedo" equilibrium dissolution temperature $T_{j}^{0}$ differs from $T_{d}^{0}$ in that it includes in its definition (through $\ln a_{j}$ ) the entropy of mixing of the various molecular weight species in solution; however, as one might expect there is very little difference (usually less than $1^{\circ}$ ) in the two temperatures.

\section{c. High Undercooling Limit}

In eq (2.25) the volume fraction $v_{2}^{j}$ of species $j$ is required. From the definition of volume fraction it can be readily shown that

$$
v_{2}^{j}=\left(M_{j} p_{j} / M_{n}\right) v_{2}
$$

where $p_{j}$ is the probability or mole fraction of species $j$ in solution, $M_{j}$ is its molecular weight $(\mathrm{MW})$ and $M_{n}=\Sigma_{j} p_{j} M_{j}$ is the number average $M W$. Note that $v_{2}^{j} \neq p_{j} v_{2}$ and that the volume fraction is a weight average property $\left(M_{w}\right)$ :

$$
M_{w}=\Sigma_{j} M_{j} v_{2}^{j} / v_{2} .
$$

In the limit of high undercoolings $B_{2}^{j} \rightarrow 0$ so that

$$
\begin{gathered}
\lambda \rightarrow \Sigma_{j} A_{1}^{j} \\
f_{j} \rightarrow A_{1}^{j} / \Sigma_{j} A_{1}^{j} .
\end{gathered}
$$

Now the forward rate constants chosen in accordance with paper II (c.f. sec. IIIa) give in the high undercooling limit.

thus

$$
A_{j}^{j} \rightarrow v_{2}^{j} / \sqrt{x_{j}}
$$

$$
f_{j}=v_{2}^{j} / \sqrt{x_{j}} /\left(\Sigma_{j} v_{2}^{j} / \sqrt{x_{j}}\right)
$$

The factor $\sqrt{x_{j}}$ arises from the reduction of polymer conformations near a crystal surface. More generally the $1 / 2$ power of $x_{j}$ is replaced by $2 \delta$ where $\delta$ has the limits of zero and $1 / 2(0<\delta<1 / 2) .{ }^{17}$ Our best estimate of $\delta$ in the equilibrium limit was $\delta=1 / 4$, however in the high undercooling limit a smaller value for $\delta$ may be more appropriate. In any event, in the absence of a surface $\delta=0$ and wo obtain

$$
f_{j} \rightarrow v_{2}^{j} / v_{2}
$$

This result is consistent with physical intuition because the probability of finding a polymer molecule at a particular place in the solution is equal to $v_{2}^{j} / v_{2}$.
Therefore, in the absence of energetic and surface effects (all polymeric species which come in contact with the crystal niche are incorporated) fractionation will occur according to volume fraction. This is a significant conclusion because as mentioned previously volume fraction is closely related to the weight average properties of the polymer and thus to a first approximation, the number average $\mathrm{MW}$ of the crystal will equal the weight average MW of the polymer in solution. Of course, we are treating the case where the composition of the solution does not change substantially during the course of crystallization. In the usual experimental range of undercoolings energetics play an important role in fractionation as will be demonstrated in section 3 .

In the above considerations we have ignored preexponential (diffusion) factors. The rate determining step is presumed to be not diffusion to the crystal surface, but rather incorporation into the growth niche. This opinion has been expressed elsewhere for dilute solution crystallization [22]. The present theory can easily accommodate diffusional factors when they exist. In the simplest approximation the diffusion constant of a polymer molecule is inversely proportional to MW and, therefore, the exponent of $x_{j}$ in eqs (2.35) and (2.36) or wherever it occurs in rate constants would change from $1 / 2$ to $3 / 2$.

\section{Results}

\subsection{Constants and Parameters}

The constants and parameters used in this study are identical to those listed in table III of paper II for polyethylene in xylene which serves as a model system. As in paper II we will employ a semi-empirical relation for the equilibrium dissolution temperature $T_{j}^{0}$;

$$
T_{j}^{0}=\left\{T_{m}^{0}(j)\left[1+. r+\left(1+r^{2}\right)^{1 / 2}\right]-29\right\} / 2
$$

where $r$ is defined by eq (2.29), Flory-Vrij values are used for $T_{m}^{0}(j)$ and the $\chi$ interaction parameter for a species of molecular weight $\boldsymbol{M}_{j}$ is the Pennings' relation

$$
\chi=0.372-25.2 / \sqrt{M_{j}} .
$$

The evaluation of the rate constants $\left(A_{0}^{j}, B_{1}^{j}\right)$ and $\left(A_{1}^{j}, B_{0}^{i}\right)$ differ from our previous evaluation of these rate constants in paper II in two ways: (1) the localization free energy $F_{I}$. now becomes equal to

$$
F_{L}(j)=k T \ln \left(v_{2}^{j} / \sqrt{x_{j}}\right)
$$

for each species $j$ and (2) $T_{j}^{0}$ replaces $T_{d}^{0}$. The replacement of $T_{d}^{0}$ by $T_{j}^{0}$ reflects the fact that the free energy associated with the mixing of the $n$ components in solution has been taken into account.

In the special case where the extended or contour length of the polymer molecule is less than the crystal thickness, $A_{0}$ and $A_{1}$ can be set equal to zero, or the extended length rather than the crystal thickness can 
be used in evaluating the rate constants with or without a correction for chain end incorporation in the crystal. Regardless of the approximation employed the result is essentially the same-negligible amounts of these short molecules are incorporated.

\subsection{Two Component System}

\section{a. Solution of $\lambda$ Equation}

A case of particular interest is the two component system, i.e., a system of solvent and two polymeric species. In this case eq (2.14) can be solved analytically with the result

$\lambda=\left\{\gamma_{1}+\gamma_{2}+\left[\left(\gamma_{1}+\gamma_{2}\right)^{2}+4\left(A_{1}^{1} B_{2}^{2}+B_{2}^{1} \gamma_{2}\right)\right]^{1 / 2}\right\} / 2$

where $\gamma_{1}=A_{1}^{1}-B_{2}^{1}$ and $\gamma_{2}=A_{1}^{2}-B_{2}^{2}$. Experimentally, one can hope to make measurements on blends of two very sharp MW fractions. The results should approximate those for two perfectly sharp MW fractions for which eq (3.4) obtains.

\section{b. Method of Calculation}

The calculational procedure for the two component system is outlined below:

(a) The mole fraction of each component in solution is fixed, as well as other parameters such as temperature and total volume fraction.

(b) A crystal thickness value is chosen and the rate constants $\left(A_{0}^{j}, B_{1}^{j}\right)$ and $\left.A_{1}^{j}, B_{2}^{j}\right)$ which are given by eqs (3.3) through (3.6) in paper II are calculated.

(c) Lambda, which is the post-nucleated growth rate of the strip, is evaluated from eq (3.4), the crystal compositions $f_{j}$ are determined from eq (2.18) and the total nucleation rate $S_{T}$ is calculated from eq (2.15). Now there are two possible types of nuclei-solution molecules or cilia. Both nucleation rates are calculated. However, each term $A_{0}^{j} \lambda /\left(B_{1}^{j}+\lambda\right)$ for cilia nucleation must be multiplied by the weighting factor $f_{j} \mid\langle j\rangle$ because the probability that a cilium belonging to molecule $j$ is available for nucleation is proportional to the fraction of $j$ molecules being incorporated into the crystal and the density of cilia along the growth strip which in turn is inversely proportional to the average spacing $\langle j\rangle$ between cilia.

(d) The growth rate $S_{T}$ passes through a maximum as a function of lamella thickness. To obtain $S_{T}(l)$, the lamella thickness $l$ is incremented in $2.5 \AA$ intervals and the above procedure (a to $\mathrm{c}$ ) is repeated at each $l$. A natural lower bound on $l$ occurs because crystal thicknesses less than a certain value $l_{c}$ are not possible since they correspond to thermodynamically unstable crystals $\left(\lambda=0\right.$ at $\left.l_{c}\right)$. For large lamella thicknesses the calculation is carried on until the nucleation rate is at least two orders of magnitude less than the maximum nucleation rate.

\section{c. Method of Averaging}

The most important quantity to be calculated is the composition of the crystallites that are formed. Although the $f_{j}$ are uniquely determined for a given crystal thickness and are independent of nucleation rate constants, theory predicts that a distribution of crystallite thicknesses may evolve. If $S_{T}\left(l_{i}\right)$ is the nucleation rate for crystallites of thickness $l_{i}$, we can define the average composition $\left\langle f_{j}\right\rangle$ (or any other property of the crystals) in the following way:

$$
<f_{j}>=\Sigma_{i} f_{j}\left(l_{i}\right) S_{T}\left(l_{i}\right) / \Sigma_{i} S_{T}\left(l_{i}\right) .
$$

On physical grounds weighting $f_{j}$ by $S_{T}$ is not strictly correct because material conservation has not been taken into account. For example, if the lateral dimensions of a crystal are proportional to $S_{T}$, then the volume or mass of the lamellar crystal is proportional to $S_{T}^{2} l$ which is the proper weighting function. However, average properties calculated with either weighting function yield similar results. Alternatively one could calculate the $f_{j}$ at the maximum value of $S_{T}$ but this procedure is not always adequate for two component systems because $S_{T}$ occasionally is slightly binodal and tends to be broad.

Either the solution molecule or cilia nucleation rates can be used to calculate average properties. In general, utilization of the cilia nucleation rate leads to better fractionation, i.e., more of the high MW component is incorporated. Since it is still unknown at this time how much each of the two nucleation rates contribute to the total nucleation rate, we have arbitrarily presented results in figures 1 thru 6 based on the solution molecule nucleation rate.

\section{d. Kinetic Phase Diagrams}

Figures 1 to 3 give some results for binary systems of polyethylene. They are kinetic phase diagrams although they differ from the usual temperaturecomposition phase diagram representations. Since the crystallization temperature is an experimentally controllable variable we have chosen to present the results for isothermal crystallization conditions.

The effect of crystallization temperature on fractionation is illustrated in figure 1 . One sees that even at $84{ }^{\circ} \mathrm{C}$ one is near the high undercooling limit given by eq (2.37). Rejection of the low MW component is enhanced as the crystallization temperature is increased. The high temperature limit which is equilibrium crystallization is of little interest since in this region the growth rates are so small that no crystals will be observed in experiments of laboratory time scale.

One sees by means of figure 1 that successive recrystallization can be used to purify a binary system. This is achieved by taking the crystals that form initially in the solution and after dissolving them in solvent, recrystallizing them and so on. A measure of the efficiency of the process is given by the initial slope of the curve. For $88^{\circ}$ the slope is about $1 / 10$ and therefore one would obtain 10 times more purity with each recrystallization. Thus, provided one is willing to spend the effort one can prepare polyethylene of arbitrary purity. 


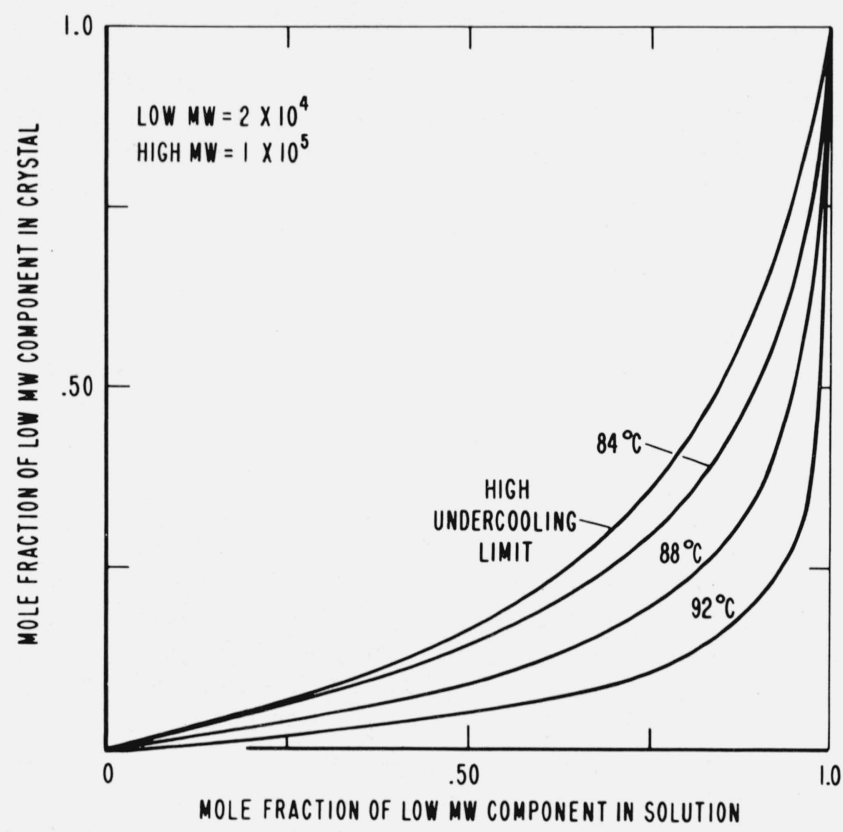

FiguRE 1. Crystal composition versus polymer solution composition for a binary PE at various crystallization temperatures.

From figure 2 one sees that the fractionation process is more efficient the larger the difference in molecular weights, and from figure 3 one sees that the process is less efficient at high molecular weights.

A general feature of all three sets of curves is that the crystal is always of higher average molecular weight than the solution from which it was crystallized. One wonders whether this is always the case. For poly-

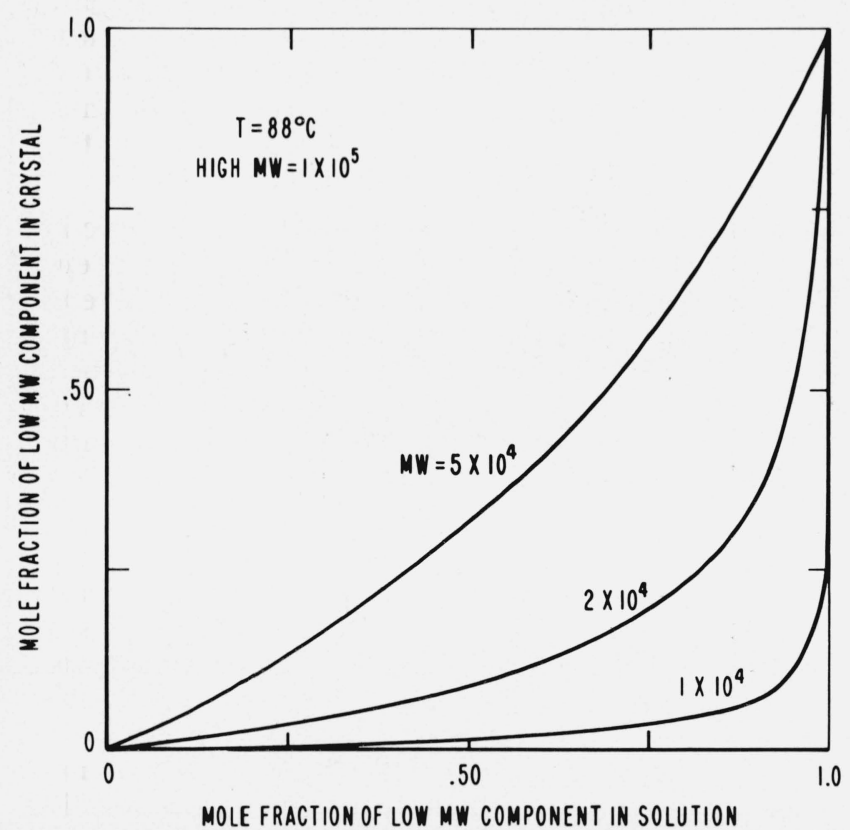

FIgURE 2. Crystal composition versus polymer solution composition for several binary PEs at a crystallization temperature of $88^{\circ} \mathrm{C}$.

The high MW component is $1 \times 10^{5}$ in all cases.

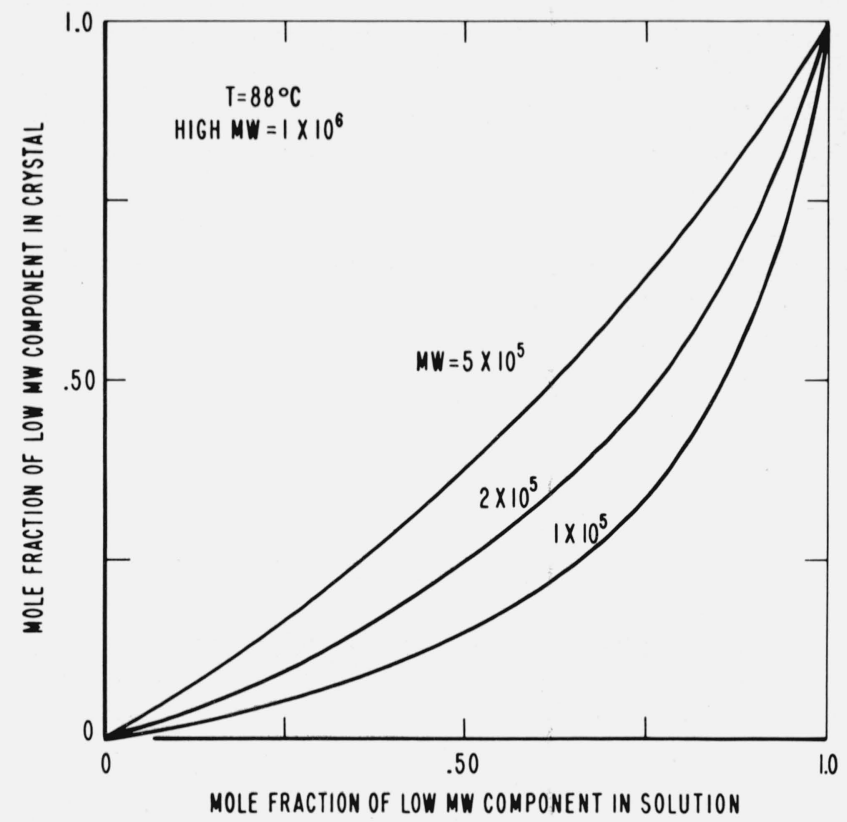

Figure 3. Crystal composition versus polymer solution composition for several binary PEs at a crystallization temperature of $88^{\circ} \mathrm{C}$.

The high MW component is $1 \times 10^{6}$ in all cases. (ca. fig. 2).

ethylene the use of eq (3.1) for the dissolution temperature guarantees that this is so. However, if for some reason a polymer system had dissolution temperatures that were constant or varied weakly with molecular weight, the question would need to be reexamined.

\section{e. Effects of Secondary Ciliation}

Another important aspect of the two component system is that it enables one to check an assumption of the present theory. Secondary ciliation has been ignored in the present theory, i.e., we have assumed that once a molecule begins folding into a growth niche it is not interrupted by another molecule. One can always go to solutions dilute enough so that this situation obtains. A theory exists for a two component system which allows for all degrees of molecular interruption during growth [25]. This theory is complicated because it involves solving a large number of nonlinear simultaneous equations. Solution of these equations for a multicomponent system is very difficult, but a binary system is reasonably amenable to analysis. In the appendix it is shown that the same fractionation is predicted from both theories at small undercoolings which suggests that neglect of secondary ciliation effects is valid also for the multicomponent system.

\subsection{Multicomponent Systems}

\section{a. Molecular Weight Distribution}

The MW distributions of commercially available linear polyethylenes are usually very broad. Weight average to number average molecular weights $\left(M_{w} / M_{n}\right)$ in the range 5 to 10 are not uncommon and log-normal 
distribution functions are often used to characterize these MW distributions [28]:

$$
\begin{aligned}
P(\log M) d(\log M)=\left(2 \pi \sigma^{2}\right)^{-1 / 2} & \exp \\
& {\left[-\frac{(\log \boldsymbol{M}-\mu)^{2}}{2 \sigma^{2}}\right] d(\log M) }
\end{aligned}
$$

If $\log M$ (common logarithm) follows a normal or Gaussian law, then $M$ is distributed according to a lognormal law:

$$
P(M) d M=[P(\log M) / M \ln 10] d M
$$

with

$$
\begin{gathered}
<M>\equiv M_{n}=10^{\mu+\sigma^{2} \ln 10 / 2} \\
<M^{2}>/<M>^{2} \equiv M_{w} / M_{n}=10^{\sigma^{2} \ln 10} .
\end{gathered}
$$

A continuous distribution function can often be accurately represented by a discrete distribution function and vice versa. In the present case it was found that a broad normal distribution can be well represent ed by a discrete distribution containing $k$ points disposed at equal intervals on the $\log M$ scale if $k$ is greater than about 25. A good representation of the continuous distribution means that the calculated $M_{n}$ and $M_{u}$ values that are obtained from the discrete distribution are close to the exact values given in eqs (3.8) and (3.9). Each interval $\Delta$ is set equal to $8 \sigma /(k-1)$ where $\sigma$ is the mean square deviation of the Gaussian distribution function (3.6). The points or intervals are distributed so that $3 / 8$ of them lie on the low MW side of the mean $\mu$ and $5 / 8$ of them lie on the high MW side of $\mu$. In the calculations presented here $k$ was set equal to 33 , i.e., fractionation is being examined in a system containing 33 different MW species of polymer. The mole fraction of species $k$ in solution is equal to $P\left(M \quad\left(M_{k+1}-M_{k-1}\right) \quad\right.$ when properly normalized.

\section{b. Method of Calculation}

The calculation procedure employed for the multicomponent system is similar to that described in section $3.2 \mathrm{~b}$ for the two component system. The mole fractions of each MW species are now determined by the log-normal distribution as discussed above. The differences in the calculations are briefly outlined below:

(a) The $\lambda$ equation, eq (2.14), is solved numerically by successive iteration beginning with the trial solution

$$
\lambda=\Sigma_{j} A_{1}^{j} .
$$

Usually 4 or 5 iterations, except very near the critical thickness $l_{c}$, secures convergence with an error of 1 part in $10^{4}$ or better.

(b) The $f_{j}$ and other properties are calculated at the maximum value of the nucleation rate rather than averaging over all nucleation rates. The nucleation rate $S_{T}$, although broad, is unimodal and average properties will not significantly differ from those computed near the maximum value of $S_{T}$.
Figure 4 is a representative example of the kind of information obtainable from theory. Curve 1 is the distribution in histogram form of the 33 different molecular weight species in solution. On the scale of the graph, 8 of the highest MW species cannot be shown because their mole fraction concentrations are so small. At high undercoolings and in the absence of energetic and diffusional effects the polymer would fractionate according to volume fraction; the volume fractions are shown in curve 2. At a crystallization temperature of $90{ }^{\circ} \mathrm{C}$ energetics are important and fractionation is enhanced. Curve 3 is the predicted distribution of MWs for a crystal formed in a solution where the distribution of $\mathrm{MW}$ s is given by curve 1 at a crystallization temperature of $90{ }^{\circ} \mathrm{C}$. The $M_{n}$ and $M_{w} / M_{n}$ for the polymer in solution are $1 \times 10^{4}$ and 9.0 , respectively. The $M_{n}$ and $M_{w} / M_{n}$ for the crystal, however, are $1.5 \times 10^{5}$ and 2.9. Will dissolution of the crystal followed by recrystallization enhance the fractionation even more? The answer is yes, but the enhancement is undramatic. Curve 4 is the result of recrystallizing from a solution where the MW distribution is given by curve 3 . The $M_{n}$ increases to about $3 \times 10^{5}$, but $M_{w} / M_{n}$ remains near 2.9 .

Figure 5 summarizes the results of many calculations. The $M_{n}$ of each MW distribution was fixed at $1 \times 10^{4}$ and the width of the distribution as measured by the $M_{w} / M_{n}$ ratio was allowed to vary. Calculations were carried out at 3 crystallization temperatures to illustrate the effect of temperature on the fractionation. As in the two component system, fractionation is enhanced as the crystallization temperature is raised.

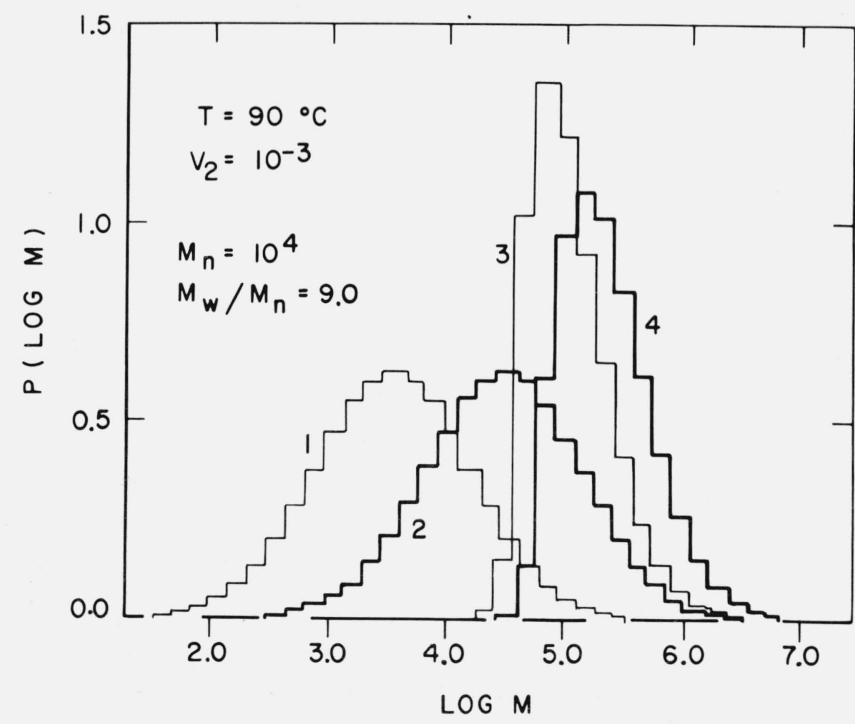

Figure 4. Fractionation histograms for a multicomponent (33) PE.

Curve 1 is a log-normal distribution of the $\mathrm{PE}$ in solution. Curve 2 is the volume fraction distribution of the polymer; in the high undercooling limit the MW distribution of the crystal that is formed would be given by curve 2 . Curve 3 is the resulting crystal $\mathrm{MW}$ distribution that obtains from curve 1 at a crystallization temperature of $90^{\circ} \mathrm{C}$. Curve 4 is the resulting crystal MW distribution that obtains from curve 3 (recrystallization). 


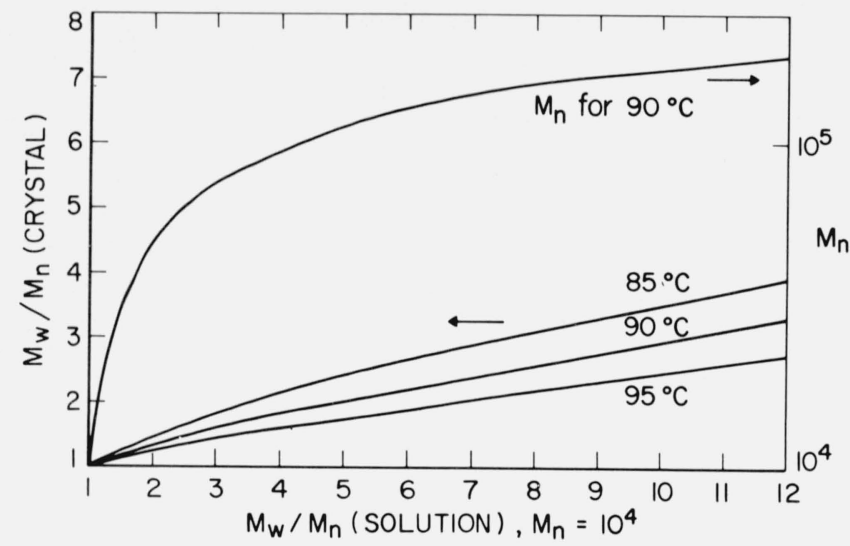

Figure 5. Molecular weight distribution $\left(\mathrm{M}_{\mathrm{w}} / \mathrm{M}_{\mathrm{n}}\right)$ in the resulting crystal versus $M W$ distribution of polymer in solution.

The $M_{n}$ of the polymer in solution is $1 \times 10^{4}$ in all cases; the $M_{n}$ of the crystal resulting from the $90{ }^{\circ} \mathrm{C}$ crystallization is also shown.

\section{d. Polydispersity Effects}

Molecular weight polydispersity can significantly affect crystal properties, especially those properties which should depend on the average MW of the polymer. In paper II we calculated the variation of crystal thickness with MW for monodisperse polymer and found that at a crystallization temperature of $90{ }^{\circ} \mathrm{C}$ a variation of crystal thickness of about $40 \AA$ occurs over a MW range of $10^{4}$ to $10^{6}$. Smaller variations are predicted for lower crystallization temperatures. Experimental attempts to observe such a variation in crystal thickness have been unsuccessful [29]. In figure 6 we have compared calculated values of lamella thickness at $90{ }^{\circ} \mathrm{C}$ for polyethylenes with various degrees of MW polydispersity. A $M_{w} / M_{n}$ ratio of 1.5 which corresponds to a relatively good fraction of $\mathrm{PE}$ reduces the variation of lamella thickness to about $20 \AA$. Fractionation during crystallization tends to mask the intrinsic dependence of crystal thickness on MW.

In general, the dependence of any crystal property on MW will be diminished by the effects of polydispersity. This includes the dependence of crystal growth rates on MW. In paper II it was shown that if cilia are the primary nucleating species in polymer crystal growth, then the dependence of crystal growth rates of polymer concentration are relatively weak. Moreover, this concentration dependence should weaken even more and eventually disappear as the MW of the polymer increases. Under normal experimental conditions the MW composition of the polymer in solution is continuously changing with time. Since the high MW components are selectively removed from the solution early in the crystallization, the $M_{n}$ of the polymer remaining in solution continually decreases. Initially the growth rate may be very weakly dependent or independent of concentration, but in later stages of growth the dependence on concentration should increase. The effect that is measured is an average over the entire range of growth which, of course, could be quite concentration dependent.

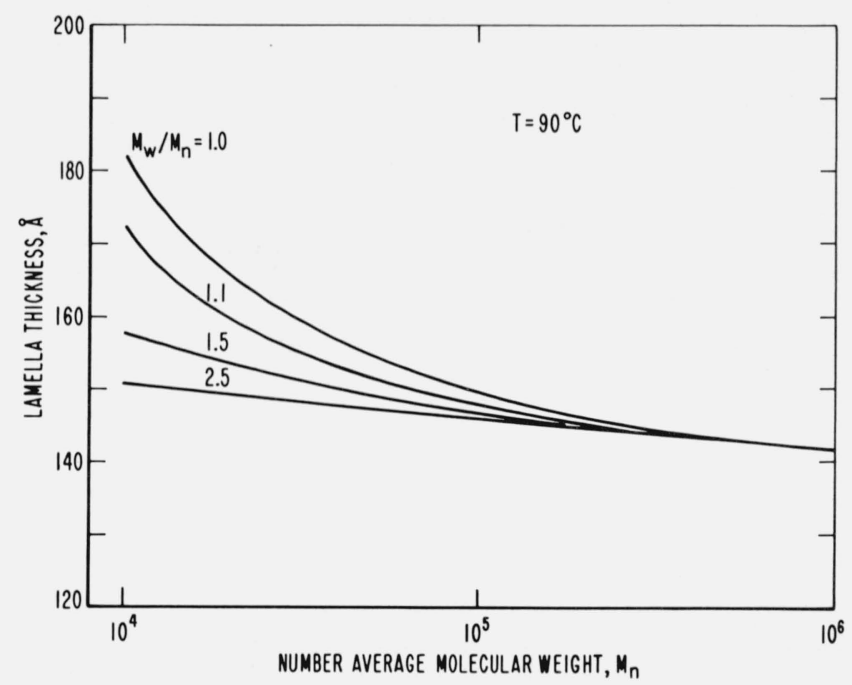

FIGURE 6. The effects of $M W$ polydispersity on crystal thickness is shown.

At crystallization temperatures lower then $90{ }^{\circ} \mathrm{C}$ the variations in crystal thickness with MW are even smaller.

It should be noted that a similar type argument cannot be put forth to justify that lamella thickness should increase as the polymer solution becomes depleted in high MW components. If, in fact, the thickness is initially established by high MW species then a persistence of that thickness over the entire range of crystal growth is to be expected. An energetically unfavorable increase in total lateral surface free energy would accompany an increase in crystal thickness (caused by the mismatch of adjacent growth strips), and although there is a favorable increase in bulk free energy associated with the increase in crystal thickness, the unfavorable increase in lateral surface free energy will be larger in the usual range of undercoolings. The net result should be that some persistence of the crystal thickness should obtain. Indeed, if this is true, then Kawai's theory $[13,14]$ of thickening of polymer crystals is no longer valid.

\section{Discussion}

\subsection{Conclusions}

A nonequilibrium theory of polymer MW fractionation has been formulated and applied to binary and multicomponent PEs crystallized from unstirred xylene solutions. We have treated the case where the composition of the polymer in solution is presumed not to vary during the course of the crystallization. From this theoretical study the following conclusions have been drawn:

(1) high MW components are selectively incorporated into the crystal;

(2) fractionation is inhanced as the crystallization temperature is raised (see figs. 1 and 5);

(3) fractionation is independent of the total polymer concentration $v_{2}$;

(4) fractionation is relatively insensitive to choices 
of the lateral and end surface free energies $\sigma$ and $\sigma_{e}$;

(5) at high undercoolings the $M_{n}$ of the crystal will be approximately equal to the $M_{w}$ of the polymer in solution from which it was formed;

(6) in binary PEs fractionation not only depends on the ratio of the MWs, but also on the absolute magnitudes of the MWs (see figs. 2 and 3);

(7) fractionation tends to mask the intrinsic dependence of crystal thickness on MW (see fig. 6);

(8) fractionation is not determined by the MW species involved in nucleation, it only depends on the rates $\left(A_{1}^{j}, B_{2}^{j}\right)$ for post-nucleation crystal growth.

The important variables that govern polymer MW fractionation are (1) the pseudo equilibrium dissolution temperatures $T_{j}^{0}$, (2) the volume fractions $v_{2}^{j}$, (3) the prefactors $1 / \sqrt{x_{j}}$ that arise due to the loss of conformational entropy of a flexible polymer near a crystal-solution interface, (4) the "dilution factor," which is to be discussed below, and (5) diffusion constants which were not explicitly considered in the present study. The variables $T_{j}^{0}$ and $v_{2}^{j}$ favor the incorporation of high MW components whereas the reremaining variables favor incorporation of low MW components.

The pseudo equilibrium dissolution temperature increases with MW because it is a function of the equilibrium melting temperature $T_{m}^{0}$ which increases with $\mathrm{MW}$ and because the $\chi$ interaction parameter which is a measure of the solubility of the MW species also varies with MW. Thus, at a fixed crystallization temperature those $\mathrm{MW}$ components with the highest dissolution temperatures have the greatest energetic driving forces for crystallization. However, there is another energetic effect which favors the incorporation of low MW components which was alluded to in section 3.2d. Incorporation of low MW polymer produces more cilia on the crystal surface; energetically a cilium contributes much less to the surface free energy than a chain fold. Therefore, dilution of the number of chain folds on the crystal surface by cilia, the "dilution factor," is energetically favorable. For PE in xylene the variation of the dissolution temperature with $\mathrm{MW}$ is sufficiently strong so that this dilution effect is overwhelmed and high MW species are favored; nonetheless, it seems possible that a polymer solvent might exist where the variation in the dissolution temperature with $\mathrm{MW}$ is sufficiently weak so that the dilution effect might dominate.

Although fractionation depends on the volume fraction distribution $\left(A_{1}^{j} \sim v_{2}^{j}\right.$ of the MW components in solution (conclusion 5), it does not depend on the total volume fraction $v_{2}$ of the polymer in solution (conclusion 3). The reason for the latter is that for dilute polymer solutions, dissolution temperatures are only weakly dependent on $v_{2}$, both theoretically [1] and experimentally $[1,2]$.

A qualifying note needs to be added to conclusion 8. The fraction of a MW species that is incorporated into the crystal is only a function of the post-nuclea- tion rate constants $\left(A_{1}^{j}, B_{2}^{j}\right)$ and the thickness of the crystal $(l)$. Since a distribution of crystal thicknesses is theoretically possible, a distribution of compositions are also possible and the average composition of the crystals formed will depend in a weak and indirect way on the nucleation rates for crystals of various thicknesses (see section 3.2b). This explains why fractionation is only weakly dependent on the value of the lateral surface free energy $\sigma$ (conclusion 4) since it only appears in nucleation rate constants.

Fractionation is also relatively independent of $\sigma_{e}$, but for different reasons. The value of $\sigma_{e}$ establishes a lower bound $l_{c}$ for the range of thermodynamically stable crystal thicknesses. The energetics which govern fractionation do not depend very much on the magnitude of $l_{c}$ or $l$, but rather on the thermodynamic stability of the crystal as measured by the difference $l-l_{c}$. Thus, the magnitude of $\sigma_{e}$ which largely determines $l_{c}$ does not play an important role in MW fractionation.

\subsection{Extensions}

Mention should be made on how the theory can be extended and improved. The first obvious extension is that nonequilibrium MW fractionation needs to be considered from stirred solutions. The situation is complicated from a theoretical point of view by virtue of the fact that the crystalline morphology is highly complex [30]. Some progress along theoretical lines has been made [2], but much work remains.

A second possible extension is that MW fractionation during crystallization from the melt needs to be considered as well as the effect of pressure on the fractionation phenomena. Although it appears reasonable to ignore diffusional and crystal thickening effects in solution crystallization, they probably play important role in melt crystallization.

In the present study we have assumed that the MW composition of the solution did not vary during the course of the crystallization. This restriction needs to be relaxed and can probably be easily accomplished by use of an adiabatic approximation.

Finally, there is a need to develop a molecular theory which will predict how the $\chi$ interaction parameter varies with MW. Fractionation is very sensitive to the variation of the dissolution temperature with MW and as was mentioned previously, the dissolution temperature depends on $\chi$. The dependence of $\chi$ on MW is probably closely associated with long range intramolecular interactions (excluded volume effects) which implies that developing a theory for $\chi$ may be as difficult as solving the excluded volume problem.

\section{Appendix: Comparison of Fractionation Effects With or Without Secondary Ciliation}

From eq (2.18) and the expressions for $\left(A_{1}^{j}, B_{2}^{j}\right)$ we obtain for the equilibrium limit $(\lambda \rightarrow 0)$

$$
f_{j}=\left(\alpha_{0}^{1} / \beta_{1}^{1}\right)_{j}(\alpha / \beta)_{j}^{v_{j}-1} \quad j=1,2
$$


where $\nu_{j}$ is the total number of folds for molecules of type $j$. The corresponding equation that obtains when secondary ciliation is allowed is given by eq (123) of reference [25]:

$$
f_{j}=\frac{\left(\alpha_{0}^{1} / \beta_{1}^{1}\right)_{j}\left[1-(\alpha / \beta)_{j}^{\nu}\right]}{1-(\alpha / \beta)_{j}} .
$$

These expressions reduce to each other in the infinite dilute solution limit. One has $\left(\alpha_{0}^{1} / \beta_{1}^{1}\right)_{j} \rightarrow 0$ and $(\alpha / \beta)_{j} \gg 1$. In the usual experimental range $(\alpha / \beta)_{j}>1$ and the expressions will still be nearly identical for large $\nu_{j}$, but not necessarily for small $\nu_{j}$.

The equivalence of eqs (A.1) and (A.2) does not mean that there is no secondary ciliation. It means rather that even the approximate treatment which ignores secondary ciliation gives the correct results for fractionation (and growth rates) even though ciliation may occur.

An estimate of the amount of ciliation can be obtained by realizing (eq 120 of ref. [25]) that for a given MW species, the fraction of it incorporated into the crystal with $k$ and only $k$ folds is proportional to $(\alpha / \beta)^{k}=B^{k}$. Thus one obtains for the expected number of folds in a polymer of length $\nu_{j}$ (maximum of $\nu_{j}$ folds) the expression

$$
\begin{aligned}
<\nu_{j}> & =B \frac{\partial}{\partial B} \ln \left[\sum_{k=1}^{\nu_{j}} B^{k}\right] \\
& =\frac{\left(\nu_{j}+1\right) B^{v_{j}+1}}{B^{v_{j}+1}-1}-\frac{B}{B-1} .
\end{aligned}
$$

In the infinite dilute solution limit $B \gg 1$ and $\left\langle\nu_{j}\right\rangle \rightarrow$ $\nu_{j}$. In the usual concentration ranges $B>1$ and $<\nu_{j}><\nu_{j}$.

\section{References}

[1] Pennings. A. J., in Characterization of Macromolecular Structure. Publication No. 1573 of the National Academy of Science, Washington. D.C., 1968, p. 214.

[2] Pennings, A. J., J. Polym. Sci. Part C. No. 16, 1799 (1967).

[3] Kawai, T., Matsumoto. T., Kato, M., and Maeda, H., Kolloid Z.Z. Polym. 222, 1 (1968).

[4] Koningsveld, R., and Pennings, A. J., Recl. Trav. Chim. PaysBas 83, 552 (1964).

[5] Sadler. D. M., J. Polym. Sci. Part A-2, 9, 779 (1971).

[6] Keith, H. D., and Padden. F. J., Jr., J. Appl. Phys. 34, 2409 (1963).

[7] Keith. H. D., and Padden. F. J., J. Appl. Phys. 35, 1270 (1964).

[8] Anderson. F. R., J. Appl. Phys. 35, 64 (1964).

[9] Kawai. T., Makromol. Chem. 84, 294 (1965).

[10] Prime. R. B., and Wunderlich, B., J. Polym. Sci. A-2, 7, 2061 (1969).

[11] Prime, R. B., and Wunderlich, B., J. Polym. Sci. A-2, 7, $2073(1969)$.

[12] Rees, D. V., and Bassett. D. C., J. Polym. Sci. A-2, 9, 385 (1971).

[13] Kawai, T., Makromol. Chem. 84, 290 (1965).

[14] Kawai, T., Kolloid Z.Z. Polym. 229, 116 (1969).

[15] Kawai, T., J. Polym. Sci. B 3, 83 (1965).

[16] Kawai, T., Makromol. Chem. 102, 125 (1967).

[17] Sanchez. I. C., and DiMarzio, E. A., J. Chem. Phys. 55, 893 (1971).

[18] Sanchez, I. C., and DiMarzio, E. A., Macromolecules 4, 677 (1971).

[19] Keller, A., Phil, Mag. 2,1171 (1957).

[20] Keller A., Rept. Progr. Phys. 31,623 (1968).

[21] Lindenmeyer. P. H., J. Polym. Sci. C 1, 5 (1963).

[22] Blundell. D. J., and Keller, A., J. Polym. Sci. B 6, 433 (1968).

[23] Hillig. W. B., Acta Met. 14, 1868 (1966).

[24] Lauritzen. J. I., Jr. DiMarzio. E. A., and Passaglia. E., J. Chem. Phys. 45, 4444 (1966).

[25] DiMarzio, E. A.. J. Chem. Phys. 47, 3451 (1967).

[26] Mullins. W. W., Phys. Rev. 114, 389 (1959).

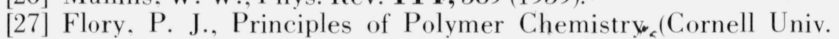
Press. Ithaca, N.Y., 1953), p. 513.

[28] Blundell. D. J., and Keller. A., J. Macromol. Sci. Phys. 2, 337 (1968).

[29] Bair, H. E., and Salovey, R., J. Macromol. Sci. Phys. 3, 3 (1969).

[30] Pennings. A. J., Van der Mark. J. M. A. A., and Kiel, A. M., Kolloid Z. Z. Kolloid Polym. 23 7, 336 (1970).

(Paper 76A3-718) 